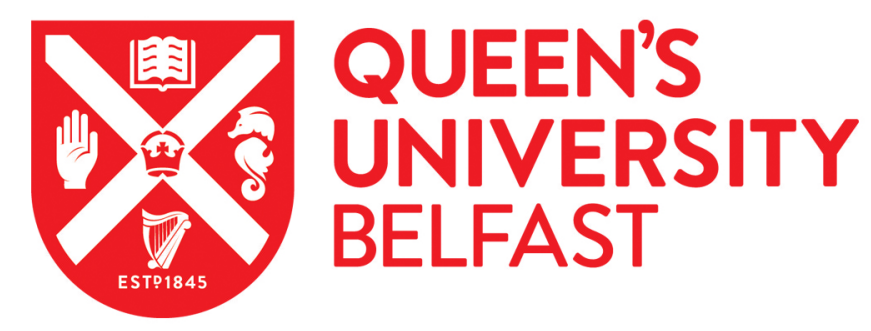

\title{
Bereavement outcomes: a quantitative survey identifying risk factors in family carers bereaved through cancer
}

Roulston, A., Campbell, A., Cairnduff, V., Fitzpatrick, D., Donnelly, C., \& Gavin, A. (2016). Bereavement outcomes: a quantitative survey identifying risk factors in family carers bereaved through cancer. Palliative Medicine, 31(2), 162. https://doi.org/10.1177/0269216316649127

Published in:

Palliative Medicine

Document Version:

Peer reviewed version

Queen's University Belfast - Research Portal:

Link to publication record in Queen's University Belfast Research Portal

Publisher rights

(C) The Author(s) 2016

\section{General rights}

Copyright for the publications made accessible via the Queen's University Belfast Research Portal is retained by the author(s) and / or other copyright owners and it is a condition of accessing these publications that users recognise and abide by the legal requirements associated with these rights.

Take down policy

The Research Portal is Queen's institutional repository that provides access to Queen's research output. Every effort has been made to ensure that content in the Research Portal does not infringe any person's rights, or applicable UK laws. If you discover content in the Research Portal that you believe breaches copyright or violates any law, please contact openaccess@qub.ac.uk. 
Final $15^{\text {th }}$ April 2016

\section{Title}

Bereavement outcomes: a quantitative survey identifying risk factors in informal carers bereaved through cancer.

\section{Introduction}

Family carers are essential to providing physical, emotional, financial and social support, and in coordinating care during the final phases of a patient's life. $1^{1,2}$ Often carers naively assume this demanding role following a 'crisis' situation or to supplement service provision to facilitate a patient dying in their preferred place..$^{3: 24}$ While enabling patients to die in their preferred place is important, it must be achieved with consideration as caring may have physical, psychological, social and financial consequences, which outlast the period of caring..$^{4: 579}$

\section{Factors influencing grief and bereavement outcomes}

\section{Place of death}

National End of Life Care policies ${ }^{3,5}$ advocate the importance of discussing and documenting patients and carers' needs and preferences, including place of care. Opinion surveys consistently show that most people would prefer to die at home, yet findings reveal a 'disconnect' between preferences and reality. ${ }^{6}$

The 'Views of Informal Carers - Evaluation of Services' (VOICES) retrospectively captured the perspective of bereaved carers in England about care delivered to adults in the last three months of life. ${ }^{7}$ Where patients had expressed a preference, the majority (81\%) preferred to die at home. However, results confirmed that only $49 \%$ actually died at home and the most commonly recorded place of death was a hospital (52\%). Grande and Ewing ${ }^{8}$ found little evidence to indicate that those who die at home have a better death than those who die in institutions or that the bereavement outcomes for relatives were better. However, Gomes et $\mathrm{al}^{9}$ reported that patients who died at home experienced similar pain levels to those who died in hospital in their last week of life, but grief was less intense for relatives of patients who died at home compared to those who died in hospital.

A study examining place of death of cancer patients across six European countries revealed England (22.1\%) and Wales (22.7\%) had the lowest percentage of cancer patients dying at home, compared 
Final $15^{\text {th }}$ April 2016

to Belgium (27.9\%), Italy (35.8\%) and the Netherlands $(45.4 \%) .{ }^{10}$ Recent figures from the General Register Office Northern Ireland ${ }^{11}$ reveal that place of death for patients with cancer in Northern Ireland during 2012 were home (37.6\%); hospital (42.1\%); hospice (10.8\%); and care home (9.4\%). The percentage of cancer patients dying at home in Northern Ireland is higher than other parts of the UK but is still lower than parts of Europe. Gomes \& Higginson ${ }^{12}$ identified that preference for a home death was positively associated with dying at home, as well as rural location; being married; living with relatives; family support and carers' preferences. Recent research ${ }^{13}$ suggests that dying at home is better than hospital for peace and grief, but requires discussion regarding preferences and relatives to be off work.

\section{Economic Deprivation}

Gomes and Higginson ${ }^{12}$ suggested that socio-economic status, marital status and age, as well as environmental factors such as healthcare and social support can influence place of death for cancer patients. The likelihood of a home death increased with long-term illnesses, low functional status, positive social and healthcare support and a preference to die at home. Murray et al ${ }^{13}$ reported inconclusive findings, particularly in the areas of functional status, social class, caregiver support, marital status and tumour type. The VOICES survey ${ }^{7}$ used postcodes recorded on the death certificate to assign each deceased person to a quintile of deprivation). Response rates varied from $37 \%$ in deprivation quintile 1 (most deprived) to $52 \%$ in deprivation quintile 5 (most affluent). Economic transition following a conjugal death is inevitable and can have a severe impact on the household, particularly when the main earner dies. ${ }^{14}$ Eligibility to bereavement benefits protects some married couples from deprivation, whereas unmarried couples may not qualify. ${ }^{15}$ The economic impact of bereavement depends on age, marital status, employment, savings and debt and although males and females may experience economic deprivation following bereavement, poverty is likely to affect females for years longer than males ${ }^{15}$.

This research, the first of its kind in Northern Ireland, identifies which factors significantly impacted on bereavement outcomes of adults who cared for someone with a cancer diagnosis, measured using the Texas Revised Inventory of Grief (TRIG). ${ }^{16}$ This was part of a wider research study which aimed to establish key factors that influence place of death. ${ }^{17}$ Capturing data from bereaved relatives is important for four reasons: it is the best way to obtain a population-based perspective on the 
Final $15^{\text {th }}$ April 2016

$\underline{\text { experiences of end-of-life care }}^{18}$; relatives are crucial in caring for and supporting patients to die at $\underline{\text { home }}^{19}$; family carers are also recipients of care; and surveying allows us to measure their grief and the perceived cost of their caring role.

\section{Methodology}

Study design and recruitment

STROBE guidelines were adhered to. The QUALYCARE-London study methodology was originally employed by Gomes et al. ${ }^{19}$ It is a population-based postal survey conducted with bereaved relatives of individuals where cancer (defined by ICD10 codes C00-D48) had been listed as the primary cause of death, where the deceased was over 18 years of age and death occurred at home, hospice, nursing home or hospital in Northern Ireland. Postal questionnaires captured quantitative data from individuals (relatives or friends) who registered the death of a person between $1^{\text {st }}$ December 2011 and $31^{\text {st }}$ May 2012. After identifying potential participants from death registrations, invitation letters and questionnaires were sent in September 2012 by the Demography and Methodology Branch (DMB) of the Northern Ireland Statistics and Research Agency (NISRA). Reply slips were included for those who did not wish to participate. A second questionnaire was sent to non-respondents six weeks after the original invitation. Ethical approval was granted by the Office of Research Ethics Committee Northern Ireland (ORECNI) in 2012 (Reference number 12/NI/0051). The return of completed questionnaires was regarded as consent to participate.

\section{Data collection}

QUALYCARE-Northern Ireland (NI) captured the socio-demographic characteristics of the deceased patient and the respondent. The questionnaire contained four measurement tools previously used in cancer and end-of-life care studies, with completion taking 60-90 minutes. Three tools collected retrospective data from the respondent about patient care: health and social care services use and informal care (Client Service Receipt Inventory) ${ }^{20}$, patient palliative outcomes in the week prior to death (Palliative Outcome Scale) $)^{21}$ and health-related quality of life (EuroQoL EQ-5D)22. The respondents' intensity of grief was captured using the Texas Revised Inventory of Grief (TRIG) ${ }^{16}$. 
Final $15^{\text {th }}$ April 2016

\section{TRIG}

The TRIG ${ }^{16}$ has been widely used in published literature. It is a 21 -item, five point self-report Likert scale consisting of two scales. TRIG I uses eight items to measure the initial grief response following the patient's death, and TRIG II uses 13 items to measure the current emotional reaction at the time of questionnaire completion. Items were developed based on the literature on normative and atypical

grief reactions. ${ }^{23}$ It has an internal consistency of 0.77 (part I) and 0.86 (part II) and a reliability of 0.74 (Part I) and 0.88 (Part II) ${ }^{24}$. When interpreting scores, the higher the score the less intense the grief reaction.

\section{Analysis}

Questionnaire data were inputted into the Statistical Package for Social Sciences (SPSS). Statistical tests included t-tests, ANOVA and correlations, which were conducted to ascertain the relationships between specific variables. Variables in the analysis included TRIG scores, respondent's relationship to the deceased, place of death, preferred place of death, employment before and after the patient's death and levels of deprivation. These variables were chosen after reviewing the literature, which highlights significant relationships between bereavement outcomes and kinship, employment, place of death and deprivation. A series of t-tests and ANOVAs were carried out to assess if there was a significant difference between mean scores for two or more groups. Where significant differences were, post-hoc Tukey analysis was used to explore between which groups the differences lie.

\section{Results}

There were 4047 cancer deaths in Northern Ireland during 2012. In September 2012, 1495 people were identified as eligible for inclusion in the study and were invited to participate. Of those eligible, 467 completed and returned questionnaires for analysis, achieving a response rate of $31.2 \%$.

\section{Respondent characteristics}

Eighty-nine percent of respondents had helped care for the deceased patient. Respondents were predominantly female (60.4\%) with a median age for respondent's of 55 years (range 25-87). Over half (52.7\%) of questionnaires were completed by the patient's son/daughter and $25.1 \%$ were completed by the patient's spouse/partner. In addition, the median time from the patients' death until 
Final $15^{\text {th }}$ April 2016

respondents completed and returned the questionnaire was 207 days (IQR 68; Range 135-276). Mean TRIG I and TRIG II scores by gender, age of respondent, relationship to the deceased, actual place of death, preferred place of death, employment and deprivation quintile are presented in Tables 2 and 3. The mean score for TRIG I was 25.3 (SD 9.4) and the mean score for TRIG II was 32.1 (SD 13.0).

The age of the patient at time of death is presented as a categorical variable with over two thirds $(67 \%)$ of deceased relatives aged over 70 years. The median age of the patient at the time of death was 75 years.

\section{Relationship}

Most respondents were sons/daughters $(53 \%)$ or spouses/partners $(25 \%)$. There was not a significant difference in TRIG I scores $(F(2,464)=2.027, \mathrm{p}=0.133)$ by relationship with the deceased relative. However, there was a significant difference between groups as determined by one-way ANOVA $(F(2,464)=4.488, p=0.012)$ for TRIG II scores (Table 3). A post-hoc Tukey test showed that the mean TRIG II scores were significantly higher (indicating lower levels of grief) for 'other relatives/friends' ( $35.69 \pm 13.4 ; p=0.012)$ compared to 'spouses/partners' $(30.8 \pm 12.5)$ and 'sons/daughters' (32.1+12.1; $\mathrm{p}=.042)$.

\section{Actual place of death}

In terms of actual place of death, $38.2 \%$ of patients died at home, $41.1 \%$ in hospital, $12.0 \%$ in a hospice and $8.7 \%$ in a care home. This finding is consistent with the actual place of death across the $\mathrm{NI}$ cancer deaths in 2012 and the non-respondents (Table 1).

There was no statistically significant difference in TRIG I or TRIG II scores by actual place of death TRIG I $(F(3,463)=2.53, p=0.056)$ and TRIG II $(F(3,463)=0.361, \mathrm{p}=.781))$.

\section{Achievement of Patient's and Respondent's preferred place of Death}

Where preferred place of death was known, $54.1 \%$ of patients and $59.3 \%$ of respondents achieved their preference. There was no significant difference between groups regarding TRIG I scores ( $F$ $(3,46)=2.011, p=0.112)$ for patients' preferred place of death. However, there was a statistically significant difference between groups as determined by one-way ANOVA $(F(3,46)=4.259, p=0.006)$ 
Final $15^{\text {th }}$ April 2016

was observed for TRIG II scores. A post-hoc Tukey test revealed that the mean TRIG II scores were significantly higher (indicating lower levels of grief) for respondents whose family members/friends did not have a preference for place of death $(40.8 \pm 14.8 ; p=0.004)$ compared to respondents who did not achieve preferred place of death (31.5 5 12.2). No significant differences in mean TRIG I or II scores were observed between the respondents who achieved preferred place of death and those who did not.

\section{Employment and respondent TRIG Scores}

A number of variables and mean TRIG I and II scores were examined in relation to employment before and after the patient died and location of household within the deprivation index. Findings revealed that $182(38.9 \%)$ respondents reported they had ceased working prior to the patient's death and $196(42.0 \%)$ had stopped work after the death of the family member/friend.

The scores at TRIG II were significantly lower for those who had stopped work (30.6) when compared with those who had continued to work (33.9) $(\mathrm{t}(417)=-2.826, \mathrm{p}=0.005)$ prior to the patient's death. The t-test did not indicate a significant difference in scores at TRIG I.

Similarly, a t-test was used to ascertain the mean differences in scores for the same groupings after the death of the patients. As above, the scores were significantly lower for those choosing to stop work (30.7) rather than continue employment (34.1) (t (452) $=-2.902, p=0.003)$. As reported above, there was no significant difference in scores at TRIG I.

\section{Deprivation and respondent TRIG Scores}

According to the quintile scoring of the deprivation variable it was clear that $40 \%$ of the cohort $(n=183)$ experienced deprivation (see Table 1).

There was no significant difference between the TRIG I scores across the deprivation groupings. However, there was a significant difference in TRIG II scores $(F(4,458)=2.655, \mathrm{p}=0.032)$. Post-hoc Tukey tests revealed that the mean TRIG II scores were significantly higher (indicating lower levels of grief) for families in quintile $4(35.2+13.6 p=0.03)$ compared to respondents in the second most deprived quintile $2(29.8 \pm 10.9)$.

\section{Discussion}


Final $15^{\text {th }}$ April 2016

This regional survey, which was conducted in Northern Ireland, shows how kinship, deprivation, employment, preferred and actual place of death impact on carers' bereavement outcomes. It provides insight into the intensity of grief in adults who cared for someone who died from cancer and may not be generalisable to other parts of Europe or bereaved carers of non-malignant diseases. Findings are based on 467 respondents, representing a response rate of $31.3 \%$. Although this response rate leaves potential for non-response bias with no data available on 1000 eligible participants who may have grieved differently ${ }^{17}$ it was similar to other UK population-based surveys ${ }^{25}$, ${ }^{26}$ where recruitment of bereaved relatives was effected by changes in data protection legislation. $\mathrm{TRIG}^{16}$ is the most commonly used self-assessment measure in the empirical literature focusing on past behaviour (Part I) and present feelings (Part II) and may be used to measure 'normal' grief ${ }^{27}$. However it has been criticised for: 'redundant' or 'benign' items ${ }^{28}$; guilt, bitterness, performance disruption and hallucinatory experiences being overlooked ${ }^{29}$; and items overlapping with measures of depression, blurring distinctiveness ${ }^{30}$ potentially limiting our insights into grief reactions of informal carers. Collecting TRIG I and II scores at one interval may have impacted on the accuracy of recall.

Previous research indicated that the nature of the relationship to the deceased was just as important as the age of the bereaved person ${ }^{31}$ and that adult children, heavily involved in caring for a dying parent experienced increased emotional distress, more health problems and strong emotional ties. ${ }^{32}$ Our findings, which were based on $53 \%$ of adult children compared to $25 \%$ of spouses/partners, with an average age of 55 years, supported that closer kinship between the survivor and the deceased had a greater impact on bereavement outcome. ANOVA and subsequent post-hoc Tukey tests showed significantly higher levels of grief for spouses/partners, sons/daughters in comparison to other relatives.

Approximately $39 \%$ of Northern Ireland's population is located in rural, less accessible communities with relatively high levels of deprivation, low levels of economic activity and greater uptake of state benefits $^{33}$. Findings indicated that $40 \%$ of our sample experienced deprivation and analysis revealed higher levels of grief in more deprived families.

The VOICES research reported that people prefer to die at home, but less than half achieve this, with just over half dying in hospital. ${ }^{7}$ Our findings revealed that $75 \%$ of patients had expressed a preference to die at home, but only $38 \%$ achieved this. Research suggests that more patients die at 
Final $15^{\text {th }}$ April 2016

home in Northern Ireland ${ }^{11}$ compared to some parts of Europe ${ }^{10}$ which may be due to higher deprivation, rural communities and lower economic activity as outlined above. Research reports more intense grief for relatives of patients who died in hospital ${ }^{9}$ and for carers whose burden increased when facilitating preferred place of death. ${ }^{34}$ Despite $62 \%$ of patients in our study dying in an institutional setting, findings showed no significant differences in bereavement scores between carers whose relative achieved preferred place of death and those who did not, and no significant differences in carer outcomes based on actual place of death.

Research suggests that carers may experience disengagement and deprivation from their normal occupation and alterations to their financial income both during and after caring, with many bereaved carers unable to return to paid employment due depression, anxiety, substance abuse or burnout. ${ }^{25,35}$ Corden et al ${ }^{15}$ indicated that how bereaved people were financially affected depended on age, marital status, employment status, savings and debt. Younger carers, whose partner had still been employed reported the largest drop in income, whereas pensioners experienced loss of occupational pensions, or loss of income from savings and reduced income pushing one in five below the official poverty line ${ }^{15}$. Although data were not captured on why respondents chose to leave work pre or post bereavement, our findings indicated that employment status was important with $40 \%$ of respondents ceasing work prior to the patient's death and $43 \%$ ceasing work after the patient's death. TRIG II scores were significantly lower in those who ceased work (indicating more intense grief), which suggests that employment acted as a protective barrier to mediate against increased grief scores, for those who remained in employment before and after caring. In cases where loss of employment and income leads to socioeconomic deprivation, it may be beneficial to ensure good financial advice, as this may reduce some problems associated with bereavement. ${ }^{14}$

\section{Conclusion}

The aim of this paper was to determine risk factors of family carers bereaved through cancer in Northern Ireland. Results highlight a range of positive and negative factors requiring consideration when designing and prioritising bereavement services. Positive factors influencing bereavement outcomes included, carers continuing in employment throughout the patient's illness and death, the patient having no preference for place of death and deaths in nursing homes. Those who ceased employment to provide end of life care for relatives with cancer had poorer bereavement outcomes 
Final $15^{\text {th }}$ April 2016

than those who remained in employment. Negative factors influencing bereavement outcomes included lower socioeconomic status and close kinship to the deceased.

Clinical implications

Cooperative employers should facilitate time off for caring and return to work after bereavement. Bereavement services should offer financial advice pre and post bereavement, prioritise lower income families, close family relatives and relatives of patients who die in hospital.

\section{Funding}

This study was funded by Cancer Focus (NI). The methodology was provided by King's College London and Cicely Saunders Institute. The N. Ireland Cancer Registry is funded by the Public Health Agency for N. Ireland.

\section{Acknowledgements}

The original methodology was developed by Gomes and Higginson at the Cicely Saunders Institute, King's College London with funding from Cicely Saunders International. 